\title{
Discrete Mode Laser Diodes with Very Narrow Linewidth
}

\section{Emission}

B. Kelly ${ }^{1}$, R. Phelan ${ }^{1}$, D. Jones ${ }^{1}$, C. Herbert ${ }^{1}$, J. O'Carroll ${ }^{1}$, M. Rensing ${ }^{1}$, J. Wendelboe ${ }^{1}$, C. B. Watts ${ }^{1}$, A. Kaszubowska-Anandarajah ${ }^{2}$, P. Perry ${ }^{2}$, C. Guignard ${ }^{2}$, L. P. Barry ${ }^{2}$ and J. O'Gorman ${ }^{1}$.

Ex-facet, free-running low linewidth $(\sim 100 \mathrm{kHz})$, single mode laser emission is demonstrated using low cost, regrowth-free ridge waveguide Discrete Mode Fabry Pérot laser diode chips. These narrow linewidths are obtained from sub $\mathrm{mW}$ emission powers and above.

Introduction: Attainment of the minimum achievable laser diode linewidth with the least associated level of device complexity and lowest component cost are critical for realising many single wavelength laser applications in optical communications, sensors and clocks. The free-running line-width reported for ordinary DFB and DBR laser devices is typically in the 1-5 MHz range which restricts their applications in coherent optical communications systems and ultra high resolution spectroscopy applications. The lowest monolithic single mode DFB laser diode emission line-width of $3.6 \mathrm{kHz}$ reported to date has been the subject of a 'hero' demonstration with a highly complex device structure emitting at extremely high powers [1].

It is well known that the Schalow-Townes (S-T) expression as modified by Henry for semiconductor lasers describes emission linewidth behaviour for diode lasers in the high power, above threshold limit [2]. While the laser emission line-width proportionality to inverse power is understood, other dependencies influence the minimum magnitude of 
the line-width other than the power emitted above threshold which merely drives a trend. Four main factors are recognized: (i) the laser $\alpha$-factor via the laser emission gain peak detuning, (ii) the unsaturated gain, (iii) the spontaneous emission coupling into the laser mode and finally (iv) the cavity length ( $\mathrm{L}_{\text {cav }}$ ) and mirror reflectivities, $\mathrm{R}_{1}, \mathrm{R}_{2}$ (via the cold cavity line-width, $\Delta \mathrm{v}_{\text {cavity }}$ or cavity finesse).

In [2] Okai et al exploited these dependencies to tailor a specific device structure to achieve the narrowest DFB emission full width at half maximum (FWHM) linewidth, $\Delta v$, reported to date from a free running laser diode of $3.6 \mathrm{kHz}$. This was achieved using: (i) an ultra-long laser diode $(\mathrm{L}=1200 \mu \mathrm{m})$ to reduce $\Delta v_{\text {cavity }}$ and compensate for the reduction in the cavity finesse arising from the low $(<<1 \%)$ DFB facet reflectivity required for stable DFB operation, (ii) a long $(360 \mu \mathrm{m})$ continuous phase shift region to reduce spatial hole-burning induced broadening and, finally, (iii) a negative laser emission line to gain peak detuning to reduce $\alpha$. Unfortunately this approach, when applied to a DFB, while successfully reducing the emission line-width, results in a very complex structure and does not lead to a very practical device configuration.

Device Operation: Discrete Mode laser diodes (DMLDs) [3] are simple, regrowth-free ridge waveguide Fabry-Pérot (FP) lasers. Single wavelength operation in DMLDs is achieved by introducing index perturbations in the form of etched features positioned at a small number of sites distributed along the ridge waveguide laser cavity. The features manipulate the FP cavity mirror loss spectrum by enhancing one FP mode and suppressing the others (Fig. 1, upper curve). The resulting optical power spectrum shows high side-mode suppression ratio (SMSR) between the selected 'discrete mode' emitting at $1546 \mathrm{~nm}$ and all other FP modes (Fig. 1, lower curve). DMLDs exhibit stable single 
mode operation over temperatures of $-40^{\circ} \mathrm{C} \leq \mathrm{T} \leq 85^{\circ} \mathrm{C}$, modulation rates up to $10 \mathrm{Gbs}^{-1}$ and resilience under strong optical feedback [4-5].

It is important to stress here that the mechanism whereby single mode emission achieved is different from that of a DFB laser. In a DMLD the facets play a more significant role in achieving lasing action by providing optical feedback thereby determining the device lasing threshold and differential quantum efficiency whereas the fundamental role of the perturbation features is to determine the single mode emission spectrum and, as we shall see here, narrow the laser emission linewidth.

Results \& Discussion: Fig. 2 illustrates overlapped DFB, DMLD and external cavity laser (ECL) laser line-width spectra measured using a delayed self-heterodyne (DS-H) line-width measuring set-up [6,7]. The fibre delay length in one arm of the set-up is 12 $\mathrm{km}$ (corresponding to a linewidth measurement resolution of $\sim 10 \mathrm{kHz}$ ). Light propagating in the short arm of the set-up modulated using an $\mathrm{LiNbO}_{3}$ phase modulator to frequency shift the detected heterodyne beat signal to $880 \mathrm{MHz}$ to enhance measurement accuracy. In Fig. 2 we plot the measured DS-H spectra for a DMLD, commercial DFB and a ECL LD we note that the operation condition (corresponding to $1 \mathrm{~mW}$ emission in Fig. 3) for all lasers are such to ensure equivalence in comparing there performance due to emission power dependence of laser linewidth plot shows the linewidth of a commercial DFBLD with $\Delta v=12.75 \mathrm{MHz}$; an ECL laser with $\Delta v=500 \mathrm{kHz}$; and a DMLD with $\Delta v=100$ kHz.. The inset of Fig. 3 shows the measured DMLD DS-H spectrum with a Lorentzian function curve-fit which yields a linewidth of $100 \mathrm{kHz}$. In Fig. 3 we plot the Lorentzian linewidth contribution versus the inverse optical power for the DMLD and the DFBLD. The DFB LD clearly shows the expected linewidth variation with power expected from 
the S-T relation. The DMLD initially at low powers has a linewidth of $1 \mathrm{MHz}$ which saturates at a linewidth around $100 \mathrm{kHz}$ at emission powers $\geq 0.5 \mathrm{~mW}$.

These DMLD linewidths are the narrowest reported from any simple monolithic short cavity laser diode.

Stable single wavelength operation and yield in DFB lasers requires a asymmetric or low reflectivity coatings of the laser facets to decouple the degenerate forward and backward running modes of the grating. This configuration can lead to large longitudinal nonuniformity in the photon density and induce either modal instability or linewidth rebroadening by the mechanism of spatial hole-burning. Consequently these design requirements limit the attainable laser linewidth and preclude DFB laser use in many applications. Overcoming these design and performance limitations (e.g. using long cavity lasers or long phase shift regions) results in impractical device configurations either in terms of their usability or manufacturability. In either case DFB devices by their intrinsic design require ultrahigh power operation to achieve a power limited, linewidth saturated regime.

In contrast to the DFB, the DMLD does not have such constraints and reaches the linewidth saturated regime at sub-mW emission levels, the mechanism for this saturation of the linewidth is unclear and is the subject of ongoing investigations. The question then arises as to the origins of the ultra narrow DMLD linewidths. For standard commercial DMLDs such as those used here with Lcav $=350 \mu \mathrm{m}$ and high facet reflectivities $\left(\sqrt{ } R_{1} \sqrt{ } R_{2}\right.$ $\cong 0.5)$, the cold cavity linewidth is $\Delta \mathrm{v}_{\text {cavity }} \cong 28 \mathrm{GHz}$. While no explicit gain peak detuning (gain peak $\lambda=1546 \mathrm{~nm}$ ) of the laser emission wavelength was designed for the 
$\mathrm{DM}$ to reduce the $\alpha$ parameter $\left(\Delta \nu \propto 1+\alpha^{2}\right)$, the DM multiple quantum well active region was fabricated in the InGaAlAs material system where due to its higher differential gain, $\alpha$ is about 2-3 compared to 5 for an InGaAsP-based DFB.

Conclusion: In this letter we have presented the first measured experimental results on the spectral line-width of a DMLD. A minimum emission linewidth of $100 \mathrm{kHz}$ was measured for the DMLD, which is 1 to 2 orders of magnitde less than the commercial DFB for equivalent optical powers.

The effect of the standard practice of increasing cavity length and detuning of the emission wavelength from the gain peak to further improve the linewidth behaviour is the subject of ongoing investigations. Future work will focus on exploiting these strategies to realise DM lasers with spectral line widths below $100 \mathrm{kHz}$ and on the extension of this ultra-narrow linewidth performance into the monolithic tuneable DMLD regime [8]. Note that none of the devices measured here were designed specifically for narrow linewidth operation. 


\section{References}

[1] M. Okai, M. Suzuki and T. Taniwatari. "Strained multiquantum-well corrugationpitch-modulated distributed feedback laser with ultranarrow $(3.6 \mathrm{kHz})$ spectral linewidth” Electron. Lett., vol 29, no 19, pp 1696-1697. 1993

[2] C. Henry, "Theory of the linewidth of semiconductor lasers," IEEE J. Quantum Electron, vol. 18, pp. 259-264, 1982.

[3] J. Patchell, D. Jones, B. Kelly, J. O'Gorman, "Specifying the wavelength and temperature tuning range of a Fabry-Perot laser containing refractive index perturbations," in Proc. SPIE. vol. 5825, pp. 1-13, 2005 (and references therein).

[4] http://www.eblanaphotonics.com/news.html

[5] C. Guignard, G. Tipaka, O. Vaudel, B. Kelly, J. Patchell, L. Barry, P. Besnard, J. O'Gorman, "Low Sensitivity to Optical Feedback and Optical Injection of Discrete Mode Lasers' IEEE Lasers \& Electro-Optics Society, pp 663 - 664, 2006

[6] T. Okoshi, K. Kikuchi, and A. Nakajama, "Novel method for high resolution measurement of laser output measurements", Electon. Lett., vol. 16, pp630-631, 1980.

[7] M. O. van Deventer, P. Spano, and S. K. Nielsen, "Comparison of DFB laser linewidth measurement techniques results from COST 215 round robin," Electron Lett, vol. 26, pp. 2018-2020, 1990.

[8] R. Phelan, W. H Guo, Q. Y Lu, D. Byrne, B. Roycroft, P. Lambkin, B. Corbett, F. Smyth, L.P Barry, J Patchell, B Kelly, J O'Gorman and J. F. Donegan, "A Novel 
Two-Section Tunable Discrete Mode Fabry-Pérot Laser Exhibiting Nanosecond Wavelength Switching" (to be published IEEE J. Quantum Electronics) 


\section{Authors affiliation:}

${ }^{1}$ B. Kelly, R. Phelan, D. Jones, C. Herbert, J. O'Carroll, M. Rensing, J. Wendelboe, C. B. Watts and J. O'Gorman (Eblana Photonics Ltd, Unit 32, Trinity Enterprise Centre, Dublin 2, Ireland)

2 A. Kaszubowska-Anandarajah, P. Perry, C. Guignard and L. P. Barry. (Research Institute for Networks and Communications Engineering, Dublin City University, Glasnevin, Dublin 9, Ireland) 


\section{Figure Captions}

Fig. 1. Upper curve: Calculated gain difference Vs wavelength for a DMLD. Lower curve: measured emission spectrum of a DMLD at a bias current of $40 \mathrm{~mA}$ showing a high side mode suppression ratio.

Fig. 2. Measured delayed self-heterodyne spectra where the offset modulation frequency $880 \mathrm{MHz}$ has been subtracted. Three spectra are shown (i) a DFB LD, (ii) a DMLD and (iii) an ECL.

Fig. 3. Comparing DFB and DMLD sources using the measured variation of emission linewidth Vs inverse power, 1/P. Inset: A typical self-heterodyne spectrum with a Lorentzian fit taken at a bias current of $40 \mathrm{~mA}$. 
Figure 1

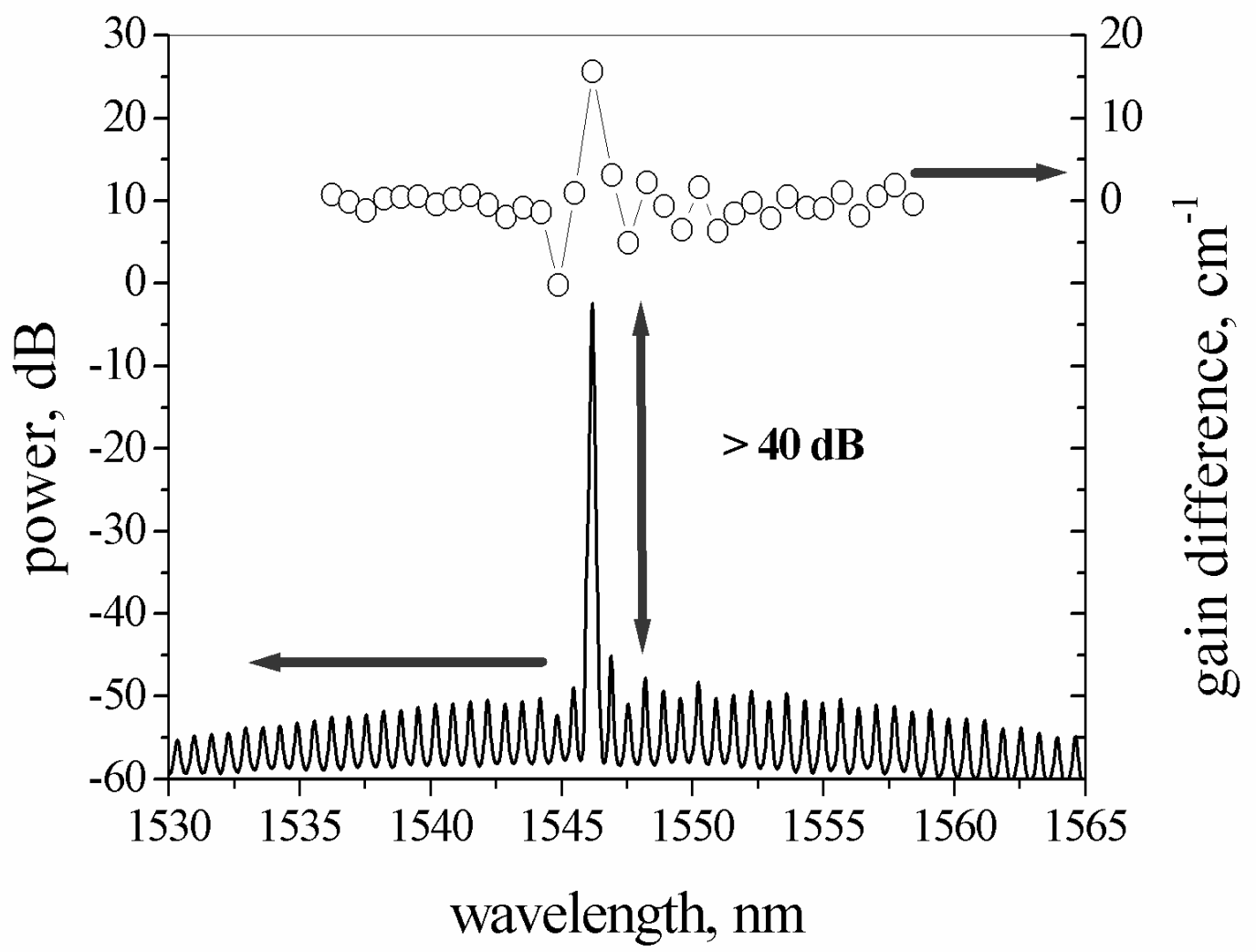


Figure 2

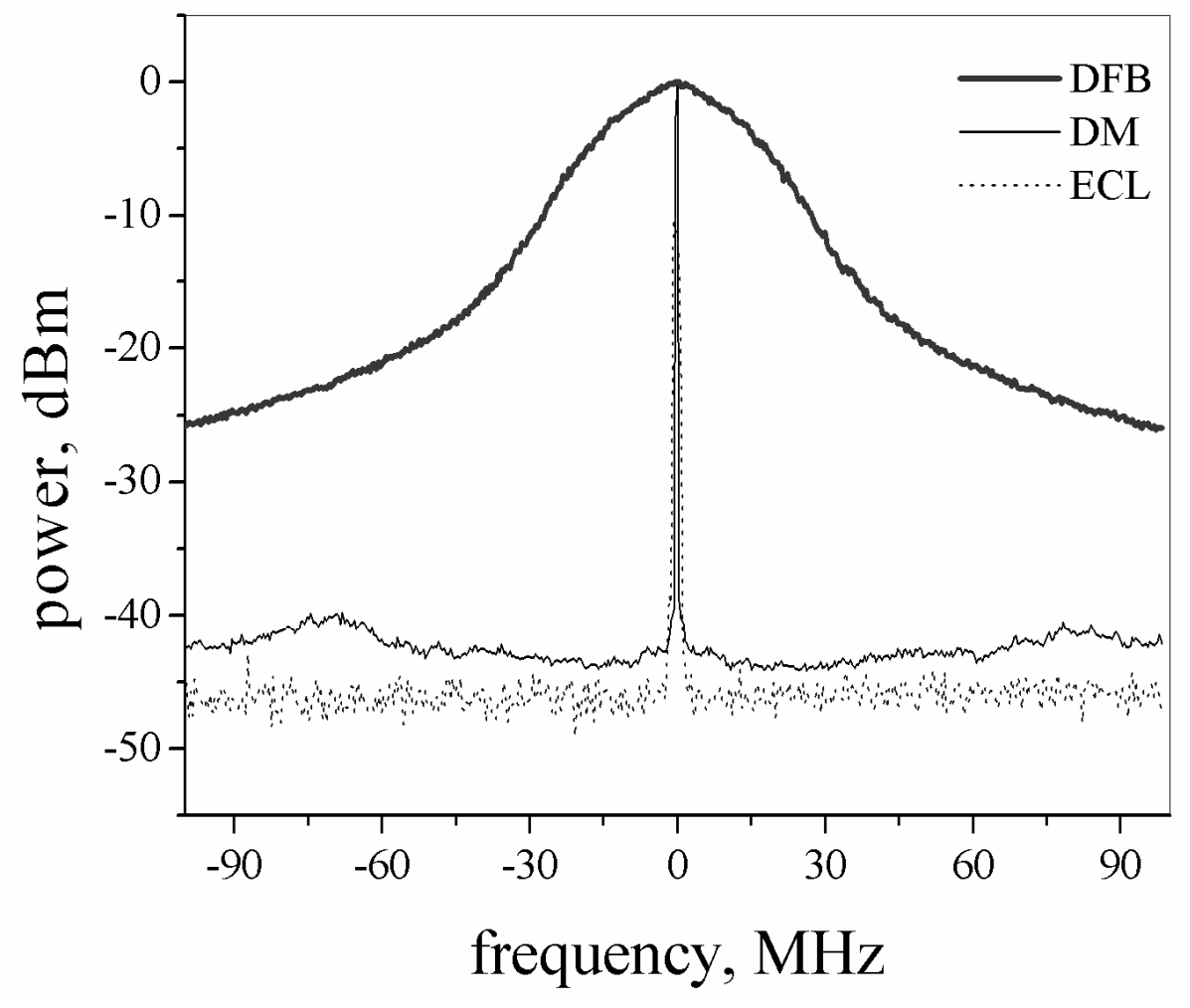


Figure 3

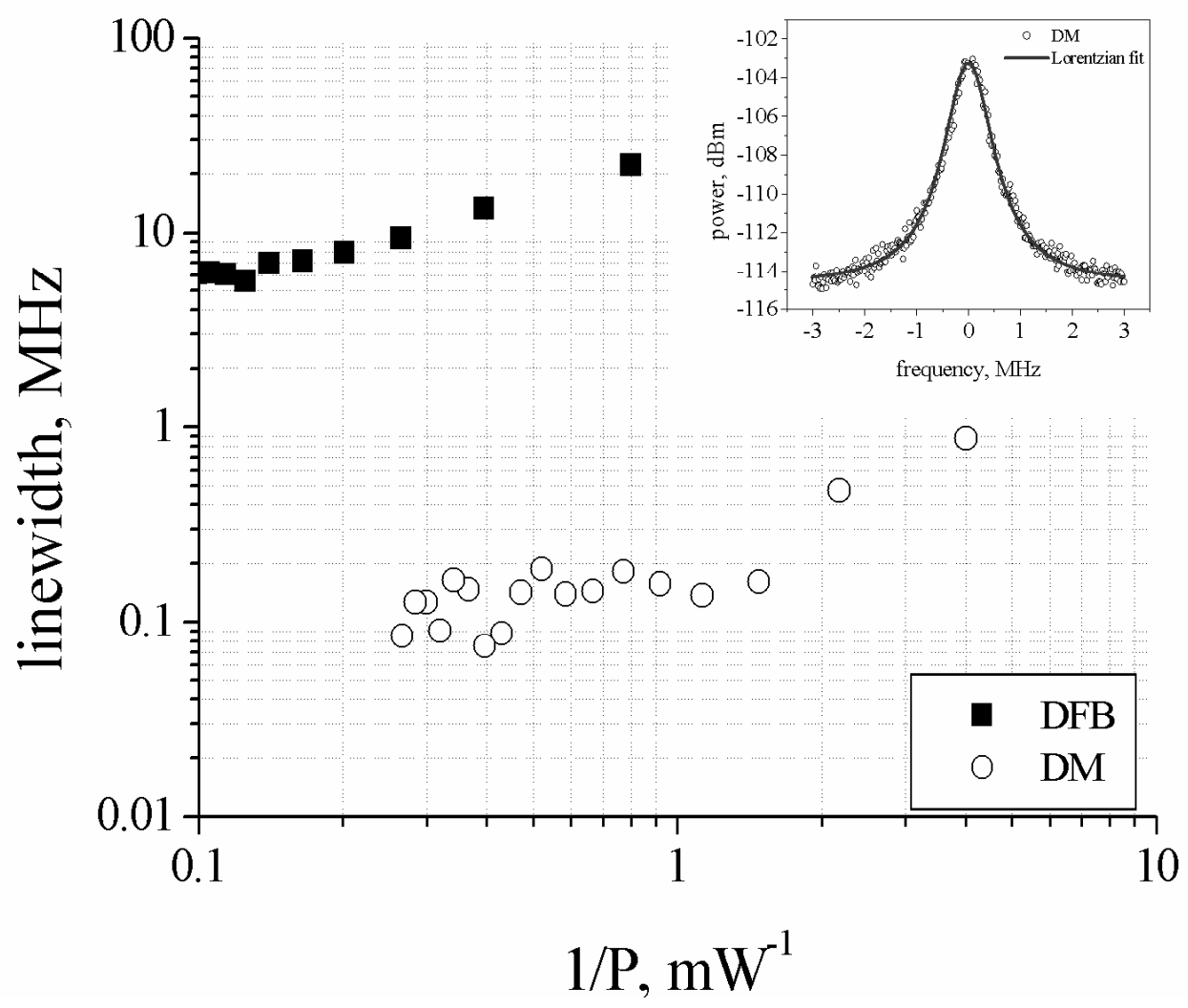

